\title{
On the Areal Random Packing
}

\author{
Masaharu Tanemura ${ }^{1}$ \\ The Institute of Statistical Mathematics, \\ 10-3 Midori-cho, Tachikawa, Tokyo, 190-8562, Japan \\ e-mail: tanemura@ism.ac.jp \\ received November 1, 2013
}

Keywords: areal packing, car parking problem, packing density

We present a new class of random packing, called an "areal random packing", in the plane. After performing computer simulations, we obtain the areal packing density through statistical analysis. One-dimensional version of areal random packing is also presented. Remarks about the connection to the random disk packing and to other processes are given.

The article is published in the author's wording.

\section{Introduction}

Random packing of objects is often seen in our daily life. Car parking problem in the street is a typical example of such random packings, but in this case, one can assume the problem is in one-dimensional space. In two-dimensional space, the random packing problem by identical disks will be a natural extension of the car parking problem into two-dimensions. Figure 1 shows a sample of this problem.

One can also consider the random packing of any objects, such as ellipses, line segments, and so on. These problems have many applications to material science, to medical science, and to other sciences.

We point out the above random packings are all consisted of the non-overlapping objects. It means that the process of random packing of objects is done by randomly putting a test object, by checking if this object overlaps existing objects, and by settling it if it does not overlap others.

In this paper, however, we present a new class of random packing problem.

\section{Areal Random Packing}

We suppose the critical size of area, instead of the non-overlapping object, which is the least accepted size of area for each generating point.

\footnotetext{
${ }^{1}$ This paper is dedicated to the upcoming 70-th birthday of Prof. Nikolay Dolbilin.
} 


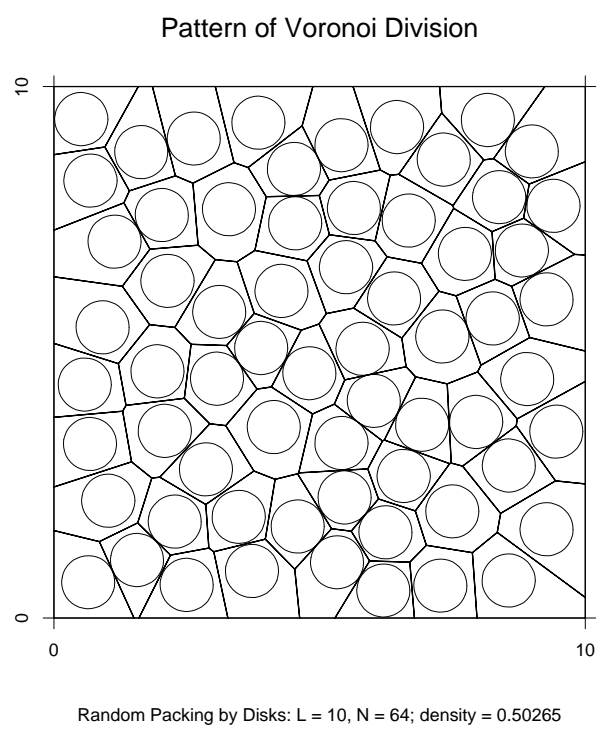

Figure 1. Sample pattern of a random packing by disks and its Voronoi division

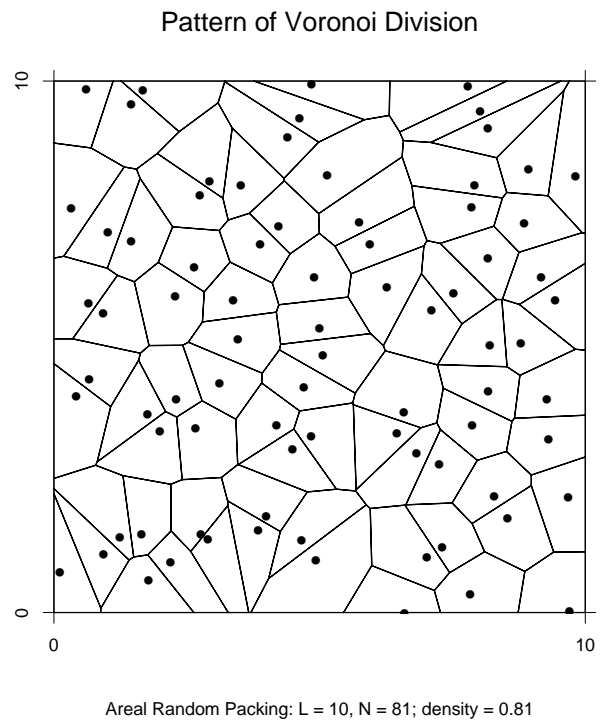

Figure 2. Sample pattern of an areal random packing

Example of the new packing process is given in Fig.2. We call this packing an 'areal random packing'. Let the whole region of the packing be a square $A=L \times L$, where $L$ is the edge length of $A$, and let $N$ be the number of points. We assume the critical value of the area allocated to each point be $a_{c}$.

A rough sketch of the procedure of our random packing is as follows:

1. Put $N \leftarrow 3$, and generate $N$ points uniformly at random inside $A$.

2. Compute Voronoi division of the point pattern. If the size of any Voronoi cell is below $a_{c}$, go to Step $\mathbf{1}$, else go to Step $\mathbf{3}$.

3. Generate a test particle $\mathbf{x}_{t}$ uniformly at random in $A$, and compute the Voronoi cell $V\left(\mathbf{x}_{t}\right)$ of the point $\mathbf{x}_{t}$. If the area of $V\left(\mathbf{x}_{t}\right)$ and the areas of 
Voronoi cells contiguous to $V\left(\mathbf{x}_{t}\right)$ are all bigger than $a_{c}$, then go to Step $\mathbf{4}$, else go to Step $\mathbf{3}$.

4. $N \leftarrow N+1$. If a specified Stopping Rule is satisfied, then Stop, else go to Step 3.

In the above procedure, a convenient Stopping Rule will be to specify the total number of test particles $n_{t}$ : if a bigger $n_{t}$ is used, the final value of $N$ will become larger. It is obvious, however, how big a value is specified to $n_{t}$, the final $N$ is limited below the value $A / a_{c}$. Then a suitable big value of $n_{t}$ is needed to avoid the waste of computational effort. In our case of Fig. $2\left(A=10 \times 10 ; L=10: a_{c}=1.0\right), n_{t}=10000$ is specified and the final value of $N$ was 81 .

\subsection{Estimation of Packing Density}

It is generally an interesting theme to estimate to what extent the whole area will be packed by the random packing process. The usual way of describing this estimate is to compute "packing density". In our case, the packing density $\rho_{A}$ is defined as $\rho_{A}=N \times a_{c} / A$. (In Fig. $2, \rho_{A}=0.81$ was obtained.)

From the above discussions, it is obvious that our random packing is a stochastic process and that $\rho_{A}$ is a random variable. Many people are often interested in the mean value of $\rho_{\infty}, \mathrm{E}\left(\rho_{\infty}\right)$, the mean packing density in the limit $A \rightarrow \infty$.

In order to estimate $\mathrm{E}\left(\rho_{\infty}\right)$, we performed computer simulation of the areal random packing for several values of $L$ with a number of independent trials $n_{s}$ for each $L$. The value $a_{c}=1$ is chosen throughout. The following table is a summary of our computer simulations.

\begin{tabular}{rrrcc}
\hline \multicolumn{1}{c}{$L$} & $n_{t}$ & $n_{s}$ & $\bar{\rho}_{L \times L}$ & $u^{-1}=L^{-1}+L^{-1}$ \\
\hline 5 & $10^{4}$ & 1000 & 0.8343 & 0.40000 \\
7 & $2 \times 10^{4}$ & 1000 & 0.8355 & 0.28571 \\
10 & $2 \times 10^{4}$ & 500 & 0.8332 & 0.20000 \\
15 & $5 \times 10^{4}$ & 500 & 0.8354 & 0.13333 \\
20 & $10^{5}$ & 500 & 0.8366 & 0.10000 \\
30 & $1.5 \times 10^{5}$ & 500 & 0.8347 & 0.06667 \\
40 & $2 \times 10^{5}$ & 200 & 0.8331 & 0.05000 \\
50 & $3 \times 10^{5}$ & 200 & 0.8324 & 0.04000 \\
70 & $2.5 \times 10^{5}$ & 100 & 0.8247 & 0.02857 \\
100 & $4 \times 10^{5}$ & 50 & 0.8222 & 0.02000 \\
200 & $2 \times 10^{6}$ & 5 & 0.8246 & 0.01000 \\
\hline
\end{tabular}

Table 1. Summary of computer simulation for the areal random packing.

In the table, $\bar{\rho}_{L \times L}$ indicates a sample mean of $\rho_{L \times L}$ and $u^{-1} \equiv X^{-1}+Y^{-1}$ is the value of the axis used for the linear regression in order to estimate $\rho_{\infty}=\lim _{X, Y \rightarrow \infty} \rho_{X \times Y}$ in the form

$$
\rho_{X \times Y}=\rho_{\infty}+a u^{-1}+o\left(u^{-1}\right) .
$$


Areal Random Packing Density: Summary

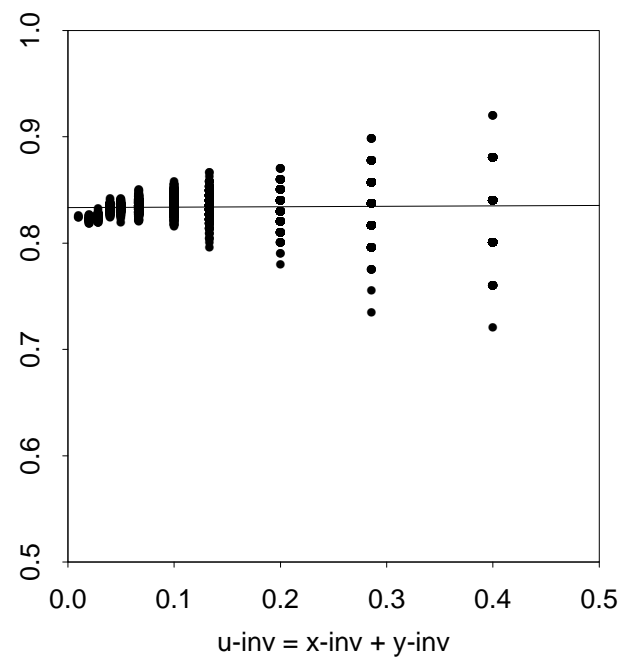

Figure 3. Summary plot of simulation and the linear regression analysis

We then performed a (weighted) linear regression of areal random packing density. Figure 3 shows the summary of our simulation and a plot of the regression.

The dots in Fig.3 indicate all of simulated value of density and the solid line in Fig.3 is an estimated regression line. From the regression analysis, we obtained for the limiting packing density $\hat{\rho}_{\infty}=0.8335$ and for the coefficient $\hat{a}=0.0040$.

Our results indicate that the areal random packing shows a packing density of about 83 percent of the area of whole region. We point out that this packing density is rather high compared with the packing density of the random packing by disks (we have obtained the value $0.5473 /(\pi / \sqrt{12})=0.6035^{2}$ for the case of disks [2]). We can consider the high packing density of areal random packing compared with the above value of identical disks might be due to the flexibility of the shape of packed objects. This fact is surmised by a comparison of Fig.1 and Fig.2, namely, the shape of Voronoi cells for disk packing is rather rotund, but the Voronoi cells for the areal packing are often flat, on the other.

\subsection{Areal Random Packing for 1-D}

It will be natural to extend the idea of areal random packing to one-dimensional space. Extension of the procedure given above to this space is rather direct and can be described as follows. Let the region of packing be a line segment $A$ whose length is $L$, and let the critical value of accepted length be $a_{c}$.

1. Put $N \leftarrow 2$, and generate $N$ points uniformly at random inside $A$.

2. Compute Voronoi division of the point pattern. If the size of any Voronoi segment is below $a_{c}$, go to Step 1, else go to Step $\mathbf{3}$.

\footnotetext{
${ }^{2}$ Here, our packing density is considered to be a 'number' density (the ratio of total number of packed points against the number of maximally packed points for the whole region), while the value 0.5473 is the 'volume' density defined as the ratio of total 'volume' of packed disks against the 'volume' of the whole region. Therefore, 0.5473 is divided by $\pi / \sqrt{12}$ (maximal 'volume' density) to get 'number' density.
} 
3. Generate a test particle $\mathbf{x}_{t}$ uniformly at random in $A$, and compute the Voronoi segment $V\left(\mathbf{x}_{t}\right)$ of the point $\mathbf{x}_{t}$. If the length of $V\left(\mathbf{x}_{t}\right)$ and the lengths of contiguous Voronoi segments are all bigger than $a_{c}$, then go to Step 4, else go to Step 3.

4. $N \leftarrow N+1$. If a specified Stopping Rule is satisfied, then Stop, else go to Step 3.

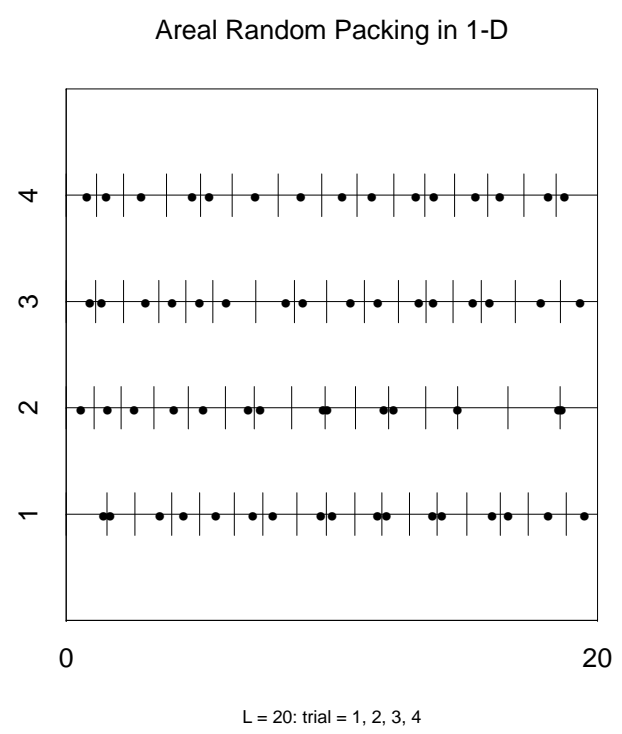

Figure 4. Sample patterns of 1-D areal random packing

Samples of the areal random packing for 1-D are given in Fig.4. In the figure, results of four independent trials are shown $(L=20)$. Vertical lines between points indicate the position of boundaries of respective Voronoi segments. From the figure, it is seen that sometimes the contiguous pair of points locate very near with each other although corresponding Voronoi segments are bigger than $a_{c}$.

As in 2-D case, in order to estimate the packing density $\rho_{\infty}$ in the limit $L \rightarrow \infty$, we performed computer simulation of 1-D areal random packing for several values of $L$ by applying the same Stopping Rule as in 2-D case ( $n_{t}$ : total number of test particles). The results are summarized in Table 2, where similar symbols are used as in Table 1 ( $a_{c}=1$ is used). Namely, $\bar{\rho}_{L}$ indicates the sample mean of $\rho_{L}$ and $u^{-1} \equiv L^{-1}$ is the value of axis used for the linear regression

$$
\rho_{L}=\rho_{\infty}+a u^{-1}+o\left(u^{-1}\right) .
$$

We performed a weighted linear regression analysis, and obtained for the packing density $\hat{\rho}_{\infty}=0.7697$ and for the regression coefficient $\hat{a}=-0.0241$.

Now, it is interesting to compare our result with the density of 'car parking problem' [1]. The problem concerns the random sequential packing of cars on the street, and a theoretical packing density is known as $c=0.74759 \cdots$. By comparing this value with our result for 1-D areal random packing, we see that the density of 1-D areal random packing is larger than $c$ but that the difference is not large in comparison with the 2-D case. 


\begin{tabular}{rrrrc}
\hline \multicolumn{1}{c}{$L$} & $n_{t}$ & $n_{s}$ & $\bar{\rho}_{L}$ & $L^{-1}$ \\
\hline 20 & $5 \times 10^{3}$ & 1000 & 0.7685 & 0.05000 \\
50 & $10^{4}$ & 1000 & 0.7698 & 0.02000 \\
100 & $5 \times 10^{3}$ & 1000 & 0.7681 & 0.01000 \\
200 & $2 \times 10^{4}$ & 1000 & 0.7700 & 0.00500 \\
500 & $5 \times 10^{4}$ & 500 & 0.7699 & 0.00200 \\
1000 & $10^{5}$ & 200 & 0.7700 & 0.00100 \\
2000 & $2.5 \times 10^{5}$ & 100 & 0.7702 & 0.00050 \\
5000 & $5 \times 10^{5}$ & 50 & 0.7700 & 0.00020 \\
\hline
\end{tabular}

Table 2. Summary of simulation for 1-D areal random packing.

\section{Remarks}

As an application of the areal random packing, we can consider the habitat of plants. It is natural to assume that an individual of plant occupies a critical size of area which is neccesary for its existence through the competition for sun light and for water or nutrition. Then, as a result of competitions among individuals, the final pattern of the plant 'territory' might look like those of the areal random packing for the asynchronous (sequential) settlement of plants. As a similar model for animal territories, we have once applied the random disk packing for the asynchronous settlement [4].

Regarding the Stopping Rule discussed in Section 2, we can devise a different rule in order to approach a random 'complete' packing. Here, a 'complete' packing is meant by the state where no further generating point can be added. In the case of random packing by disks, we have presented an algorithm called CPA (Complete Packing Algorithm) [2], which substantially uses the Voronoi vertices for the search of residual spaces for additional disks. In our case, in order to build the algorithm corresponding to CPA, we inspected several samples which are assumed to be near the complete packing. As a result we arrived at the following statements by which we are able to attain CPA. Let us assume the current packing state under consideration is approaching a complete packing in 2-D areal random packing. Then

- If there is a point $\mathbf{x}_{i}$ whose Voronoi cell area $\left|V\left(\mathbf{x}_{i}\right)\right|$ satisfies $\left|V\left(\mathbf{x}_{i}\right)\right| \geq$ $2 a_{c}$, then an acceptable point $\mathbf{x}$ lies near $\mathbf{x}_{i}$.

- If there is a contiguous pair of points $\mathbf{x}_{i}$ and $\mathbf{x}_{j}$ satisfying $\left|V\left(\mathbf{x}_{i}\right)\right|+$ $\left|V\left(\mathbf{x}_{j}\right)\right| \geq 3 a_{c}$, then an acceptable point $\mathbf{x}$ lies near the common edge $e(i, j) \equiv V\left(\mathbf{x}_{i}\right) \cap V\left(\mathbf{x}_{j}\right)$.

- If there is a contiguous triplet of points $\mathbf{x}_{i}, \mathbf{x}_{j}$ and $\mathbf{x}_{k}$ satisfying $\left|V\left(\mathbf{x}_{i}\right)\right|+$ $\left|V\left(\mathbf{x}_{j}\right)\right|+\left|V\left(\mathbf{x}_{k}\right)\right| \geq 4 a_{c}$, then an acceptable point $x$ lies near the common vertex $v(i, j) \equiv V\left(\mathbf{x}_{i}\right) \cap V\left(\mathbf{x}_{j}\right) \cap V\left(\mathbf{x}_{k}\right)$.

By taking into consideration these statements for the algorithm, we will be able to devise a CPA as a next step of the present study, and we will get a complete packing efficiently without generating much test particles. Then the density of areal random 'complete' packing will become slightly bigger than the present result. One-dimensional 
version of CPA for the areal random 'complete' packing will also be devised by suitably reforming the above statements.

We finally remark on the shape of Voronoi cells of our areal random packing. As mentioned above, we noted a qualitative difference of the Voronoi cell shapes between the areal and the disk random packings. Then, it will be an interesting problem to quantitatively compare the Voronoi cell shapes among different random processes. Regarding this, we have done an analysis of large scale computer simulation of Poisson Voronoi cells [3] and presented several formula for statistical distribution of the Voronoi cell shapes. These formula will be useful for our present problem.

\title{
References
}

1. Rényi A. On a one-dimensional problem concerning random space filling. Publ. Math. Inst. Hungar. Acad. Sci., 3: P. 109-127, 1958.

2. Tanemura M. On random complete packing by discs. Annals of the Institute of Statistical Mathematics, 31: P. 351-365, 1979.

3. Tanemura M. Statistical distributions of Poisson Voronoi cells in two and three dimensions. Forma, 18: P. 221-247, 2003.

4. Tanemura M. and Hasegawa M. Geometrical models of territory - I. Models for synchronous and asynchronous settlement of territories. Journal of Theoretical Biology, 82: P. 477-496, 1980.

\section{О региональных случайных упаковках}

\author{
Масахару Танемура \\ Институт статистической математики, \\ 10-3 Мидори-чо, Тачикава, Токио, 190-8562, Япония
}

Ключевые слова: региональные упаковки, проблема парковки, плотность упаковки

Мы вводим новый класс случайных упаковок, который называется "региональные случайные упаковки". После компьютерных симуляций, с помощью статистического анализа нами получена плотность региональных случайных упаковок. Также мы исследуем одномерный случай региональных случайных упаковок. Приведены некоторые замечания о связи случайных упаковок дисков с другими процессами.

Статья публикуется в авторской редакции.

\section{Сведения об авторе: \\ Масахару Танемура,}

Институт статистической математики, профессор 